Published Online: 4 September 2018

Proc Indian Natn Sci Acad 84 No. 4 December 2018 pp. 913-922

(C) Printed in India.

DOI: $10.16943 /$ ptinsa/2018/49448

Review Article

\title{
Food Fortification: A Public Health Approach to Bridge the Gap Between Requirement and Intake of Micro-nutrients
}

\author{
PREMA RAMACHANDRAN* \\ Director, Nutrition Foundation of India, Qutab Institutional Area, New Delhi 110 016, India
}

(Received on 22 June 2018; Accepted on 04 July 2018)

\begin{abstract}
Food fortification aims at bridging the gap between dietary intake and nutrient requirement without any dietary modification by fortifying commonly consumed food stuff with appropriate amount of nutrients. Food fortification is the preferred public health intervention to combat nutrient deficiencies due to low nutrient intake in large segments of the population, high prevalence of micro-nutrient deficiency and well documented adverse health consequences of such deficiency. Appropriate technologies have to be developed for fortifying the chosen food stuff with chosen micro-nutrients and the product has to be tested to assess safety, efficacy and acceptability. This has to be followed up with research studies to document the benefits of the usage of these fortified food stuffs. Once products with proven benefit are identified, production has to be scaled up and the fortified food has to be supplied through appropriate programmes. All public health food fortification programmes should be monitored using process and impact indicators. The data should be reviewed periodically for early identification of emerging problems and initiation of mid-course corrections in the programme.

It is well known that multiple micro-nutrient deficiencies co-exist. Efforts have therefore been made to fortify multiple food stuffs with multiple micro-nutrients even though there were technological challenges and cost implications. Review of available data from global studies indicates that such an approach may not have the intended benefit of reducing multiple micro-nutrient deficiencies. In persons who are already consuming the required amount of micro-nutrients, the consumption of multiple food stuffs fortified with multiple nutrients may lead to higher than required intake of these. Excessive intake or imbalance of intake between nutrients may have adverse health consequences.
\end{abstract}

Keywords: Food Fortification; Iron; Folic Acid; Vitamin B12; Vitamin D; Vitamin A

\section{Introduction}

Micro-nutrient deficiencies are major public health problems that affect all segments of the population both in developed and developing countries. A third of the global population and majority of Indians suffer from micro-nutrient deficiencies. Six decades ago India had recognized that iodine deficiency resulting in cretinism and goitre, Vitamin A deficiency leading to blindness in young children and iron and folic acid deficiency resulting in anaemia with adverse health consequences to the mother child dyad were major public health problems. The country embarked on intervention programmes to combat these deficiencies through dietary diversification, micro-nutrient supplementation and food fortification.
The National Goitre Control Programme (NGCP) initiated in 1962 was the first food fortification programme to combat iodine deficiency disorders. During the initial three decades, coverage under the programme was very low. During the 1980 s and the 1990s, production and distribution of iodised salt improved. But even in 2006, National Family Health Survey (NFHS) reported that household use of iodised salt was only 50\% (NFHS-3 2006). In 2007, the Government of India mandated that only iodised salt should be made available for human consumption. During the next decade, there was a steep increase in household access to iodised salt. Currently, over $90 \%$ of households use iodised salt (NFHS-4 2015). The success in rapid upscaling of the household access 
to iodised salt in the last decade has provided an impetus to food fortification programmes for combating other micro-nutrient deficiencies in India.

Through the fortification of appropriate food stuffs, it is possible to increase the intake of specific micro-nutrients without any dietary modification and sustain it over a long period of time, so that there is progressive reduction in micro-nutrient deficiencies at population level. Food fortification has become a viable, sustainable, effective and relatively inexpensive strategy to combat widespread micro-nutrient deficiencies. But care has to be taken to ensure that the technology is not used to fortify multiple food stuffs with multiple micro-nutrients because excessive intake of nutrients or imbalance between nutrients can have adverse health consequences. This article reviews the food fortification strategy to combat public health micro-nutrient deficiencies and their potential impact on the country's progress towards the targets set for 2030 under the Sustainable Development Goals (SDG).

\section{Food Security and Food Fortification}

Global and Indian experience has shown that dietary diversification and nutrition education are the two long term sustainable methods for the prevention of micronutrient deficiencies, ensuring that "all people, at all times, have physical and economic access to sufficient, safe and nutritious food that meets their dietary needs and food preferences for an active and healthy life" (FAO 1996). Data from survey conducted by National Nutrition Monitoring Bureau (NNMB 2012) have shown that currently a majority of Indian households are food secure (adequate energy intake), but vegetable intake in most households is low. This is the major factor responsible for the high prevalence of anaemia and vitamin A deficiency in the country especially amongst the poorer segments of our population.

The three-pronged strategy for combating micro-nutrient deficiencies, includes dietary diversification, nutrient supplementation and food fortification. Nutrition education aimed at improving dietary diversification and increased intake of vegetables rich in micro-nutrient content is an important component of all nutritional interventions. But it can succeed (especially among the poorer segments of population) only when vegetables are available throughout the year at an affordable cost. Interventions under the National Horticultural Mission (NHM 2016) have led to a substantial increase in vegetable production in the last decade. However, per capita consumption still remains low, partly because of bottlenecks in access and partly because of the high cost of the vegetables. Supplementation programmes to combat anaemia and vitamin A deficiency both in India and elsewhere, have shown that it is difficult to sustain long-term nutrient supplementation programmes for large population groups. Given these circumstances food fortification may be an effective, inexpensive and sustainable measure for combating widespread micro-nutrient deficiencies especially among the poorer segments of population.

\section{Types of Food Fortification}

Food fortification is "the practice of deliberately increasing the content of an essential micro-nutrient (vitamins or minerals) in a food stuff so as to improve the nutritional quality of the food supply and to provide a public health benefit with minimal risk to health" (WHO 2006). The terms 'food enrichment' and 'replacement fortification' refer to the addition of micro-nutrients which are lost during processing of a food e.g., vitamin A from milk during preparation of low-fat milk.

There are three major categories of food fortification: (a) fortification as a public health intervention to bridge the gap in nutrient intake across population groups; (b) targeted food fortification aimed at bridging the nutrient gaps in specific "at risk" population groups with nutrient deficiencies and (c) market driven fortification aimed at increasing product appeal without nutritional rationale.

\section{Public Health Food Fortification}

Involves identifying micro-nutrient deficiencies of public health importance and evolving and evaluating appropriate technologies for fortification of widely consumed food stuffs with these micro-nutrients. Food stuff that are to be fortified for public health intervention and the amount(s) of the nutrient(s) used for fortification should be chosen with care because the product may be widely used by large segments of population, who may continue to take the fortified food (sometimes without even knowing that it is fortified) 
for long periods of time without adequate monitoring, supervision or evaluation. Initiating public health food fortification programmes is a long and sometimes very tedious process. Major steps to be followed in the process include:

- $\quad$ obtaining data on the intake of each nutrient in specific population groups and identifying the proportion of the population in whom intake is inadequate

- identifying nutritional deficiencies of public health importance

- $\quad$ identifying the most commonly consumed food stuffs in the population and amount of the food stuff consumed daily

- determining the amount of nutrient to be added to the food stuff for fortification by assessing:

$>\quad$ the magnitude of gap in nutrient intake in the 'at-risk' population (with high levels of nutrient deficiency) and the amount of nutrient required to bridge the gap between requirement and intake in this group (Fig. $1)$.

$>$ the current consumption levels of the highest consumption group and the gap between total intake (food + fortification) in this group and the Tolerable Upper Limit (TUL) (Fig. 2).

- evolving appropriate technologies for fortifying the chosen food stuff with the chosen micronutrient(s), and assessing safety and efficacy of the fortified product;

- undertaking studies to assess the organoleptic properties, acceptability and use effectiveness of fortified food stuff(s);

- $\quad$ assessing the feasibility of scaling up production and working out the cost of expanding the fortification programme;

- $\quad$ scaling up production and improving access to the fortified food, beginning with vulnerable segments of population who are receiving food supplements; widening access in a phased manner through Public Distribution System (PDS) and then the open market.

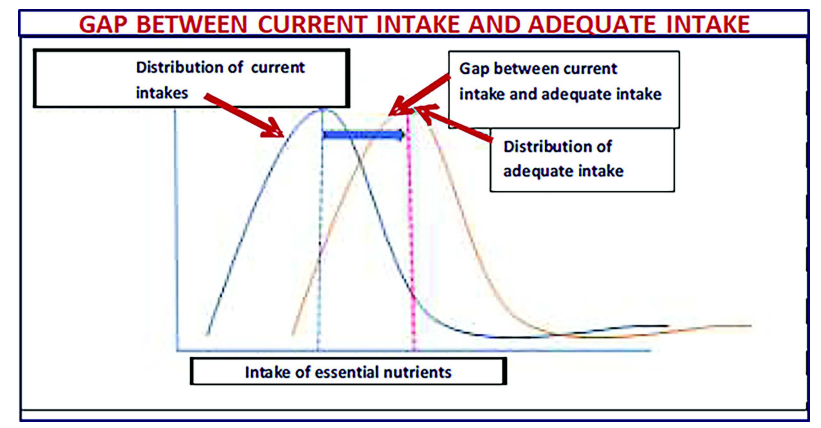

Fig. 1: Gap between intake of nutrients and nutrient requirement (copy right permission obtained from NFI Bulletin)

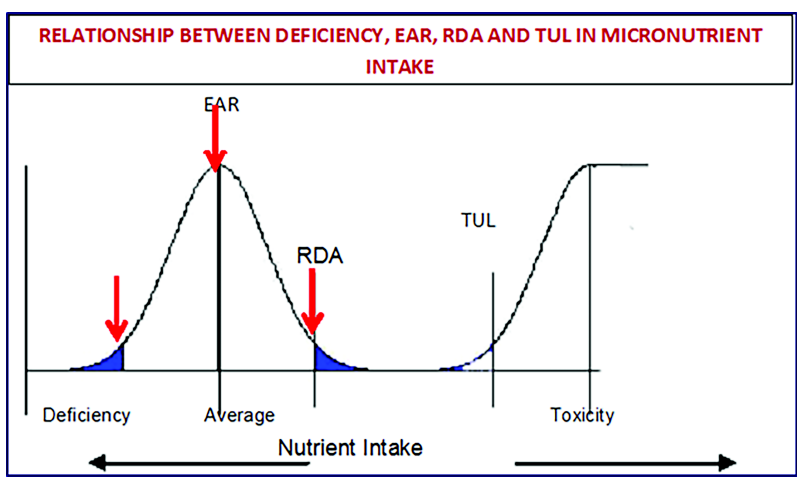

Fig. 2: Relationship between Estimated Average Requirement (EAR), Recommended Dietary Allowance (RDA) Tolerable Upper Limit (TUL) (copyright permission obtained from NFI Bulletin)

- $\quad$ simultaneously initiating awareness programmes on the availability and benefits of use of the products so as to generate a demand for them.

Ideally, the food stuff should be fortified with the selected nutrient to a level which meets all the following criteria:

- daily consumption (of the fortified food plus other regular food) meets the requirement of the nutrient in those with low intake;

- the total intake of the nutrient is not much higher than Recommended Dietary Allowance (RDA) for that nutrient (Fig. 1); and

- the total intake is substantially lower than Tolerable Upper Limit (TUL) for those with adequate intake from their regular food alone (Fig. 2).

It is relatively easy to determine the required 
level of fortification with nutrients in situations where the fortification is done in only one food stuff, the amount of nutrient required to bridge the gap in the low-intake group is small, and the difference between the total intake of nutrients from normal plus fortified food and the TUL is large. Fortification of common salt with iodine to combat IDD had all these advantages. It is difficult to embark on fortification when the gap between nutrient intake and RDA is large and the gap between RDA and TUL is small. The RDA for iron for men is $17 \mathrm{mg} /$ day but for pregnant women it is $38 \mathrm{mg}$ /day and the TUL is 45 $\mathrm{mg} /$ day. If multiple food stuffs are fortified with iron, and some segments of the population consume more than one food product fortified with iron, the total intake of iron from their normal food plus multiple fortified foods may cross the TUL even though fortification of any one food stuff was only about $30 \%$ of the RDA. On the other hand it is possible that even though the amount of nutrient used for fortification is adequate to bridge the nutrient gap when the recommended quantity of food stuff is consumed, the gap is not bridged in actual practice because the amount of the food stuff consumed is lower in some segment of the population; fortification of milk with vitamins $A$ and $D$ falls under this category.

\section{Mandatory Fortification}

It is considered only in those rare occasions when there is a major public health problem due to micronutrient deficiency, across all segments of the population, which cannot be tackled through a foodbased approach. In the Indian context iodine and iron qualify under these stringent criteria. Adequate production, transport and marketing of the fortified food should be ensured before considering mandatory fortification. Over four decades have elapsed between the initiation of National Goitre Control Programme (1962) and implementation of mandatory fortification of salt with iodine (2007).

\section{Targeted Food Fortification}

It has been used mainly to combat micro-nutrient deficiencies in specific groups such as infants and young children (who do not consume adequate micronutrients) by fortifying complementary foods with one or more micro-nutrients that are deficient in the food.

\section{Market-driven Fortification}

It is the fortification of several food stuffs often with multiple micro-nutrients without any specific nutritional rationale. This is being done mainly to increase the market share of the product by claiming nutritional and health benefits, so that segments of the population who are health conscious but perhaps not nutritionally well-informed may purchase and use these products.

\section{India's Universal Salt Iodisation Programme: Lessons Learnt}

Iodine deficiency disorders (IDD) are global public health problems (Fig. 3) (de Benoist 2008). As IDD are caused by deficiency of iodine in water, soil and foodstuff, they affect all socio-economic groups in defined geographic areas. Manifestations of iodine deficiency depend upon the time, severity and duration of the deficiency. Intrauterine exposure to maternal iodine deficiency results in creatinism. Less severe manifestations of intrauterine iodine deficiency are intellectual disability, psychomotor defects, and hearing and speech impairment. Iodine deficiency is a major cause of preventable mental retardation and brain damage in children. In women iodine deficiency can results in higher rates of infertility, abortion and stillbirth. In all age and both sex groups iodine deficiency can result in goitre and hypothyroidism. Switzerland and U.S.A initiated salt iodization programmes in 1920s to combat endemic goitre. Over the next decade there was a steep decline in prevalence of goitre and IDD. This gave an impetus to use of salt fortification as the strategy to combat IDD.

Goitre has been well recognized as a public health problem in the sub-Himalayan population.

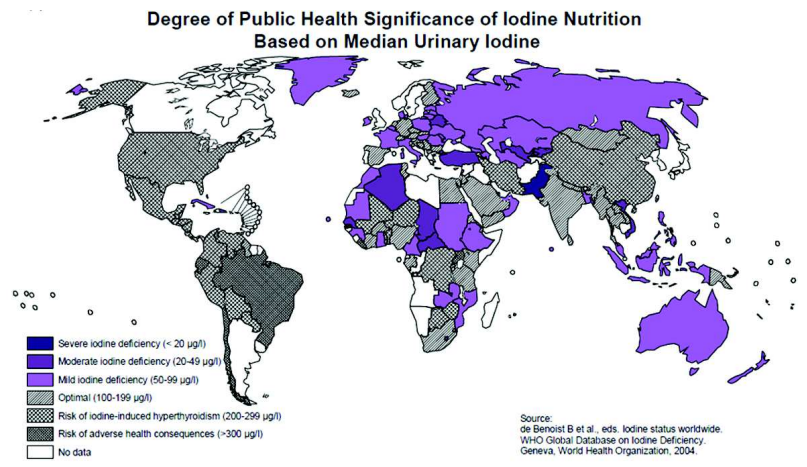

Fig. 3: Median Urinary iodine levels in different countries (de Benoist-2008) 
Successful demonstration in the Kangra valley that use of iodised salt resulted in reduction in goitre led to the initiation of the National Goitre Control Programme (NGCP) in 1962. The programme provided iodized salt to the population living in the sub-Himalayan goitre belt. Supply of salt was erratic and households continued to use the cheaper non-iodized salt; as a result, there was no substantial reduction in IDD. In the 1980s DGHS/ICMR/state IDD cells (DGHS 2018) conducted surveys in districts across the country and reported that there are pockets of iodine deficiency in all the States. In view of this, a decision was taken to iodize all the edible salt for human consumption in the country. As all IDD are to be combated with salt iodisation programme it was decided to rename NGCP as the National Iodine Deficiency Disorders Control Programme (NIDDCP) (DGHS 2018).

Adequate production of iodized salt in the country is an essential pre-requisite for improving household access to iodised salt. Efforts to scale up iodised salt production and opening salt production to private sector led to the installed capacity getting scaled up to over 10 million metric tons per year. The Salt Commissioner's office was given the responsibility of testing salt for adequacy of iodisation and certifying it. The certified salt was transported on priority and at a subsidized cost by Railways. In the seventies and eighties, most of the iodine fortified salt was not powdered; therefore there was a risk of uneven mixing of iodine. Most of the salt was transported in jute sacks piled in open rail rakes and exposed to the rains and hot sun. At the retail level in many shops, the sacks were left open outside the shop exposed to the sun and rain. In the kitchens salt was stored in open pots near the kitchen fire. As a result, the iodine content of salt at retail and household levels was substantially lower than the original content immediately after manufacture. Taking these factors into account, the standards for salt iodisation at the manufacturers' level was set higher at $30 \mathrm{ppm}$, so that the iodine available at the retail/household level would be about $15 \mathrm{ppm}$. This practice was considered appropriate as the $\mathrm{WHO}$ had indicated that safe level of use of iodine can be upto $1 \mathrm{mg}$ /day (WHO 2006).

The population in the goitre belt were familiar with the severe adverse consequences of IDD and so accessed available iodised salt (household salt use was $80 \%$ in NFHS2 1999). Populations in the states that had not experienced the adverse clinical consequences of IDD continued to purchase the cheaper non-iodised salt. Government of India advised all the states to ensure mandatory iodisation of salt meant for human consumption but in 2000, the ban on the sale of non-iodised salt was lifted. This led to a reduction in households using iodized salt (NFHS-3 2006). Government of India reinstated the ban in 2005 and over the last decade there has been improvement in household use of iodised salt. Currently, over $90 \%$ of the households in the country use iodised salt (Fig. 4) (NFHS 4 2015). This has demonstrated that, once the production, distribution, sale processes have been streamlined and awareness has been generated, it is possible to increase the household use of iodised salt by nearly $50 \%$ within a decade.

\section{Emerging Issues Regarding Fortification of Salt with Iodine}

Over the last two decades there has been an increase in the use of powdered salt for iodisation ensuring more uniform mixing of iodine in salt. A majority of manufacturers now pack iodised salt in $1 \mathrm{~kg}$ polypacks. At household level (in many urban areas) the salt is stored in the polypack or in a plastic bottle. As a result, the iodine loss from the salt has been minimized. It is essential to collate and review data available with the Salt Commissioner's office, the Ministry of Health

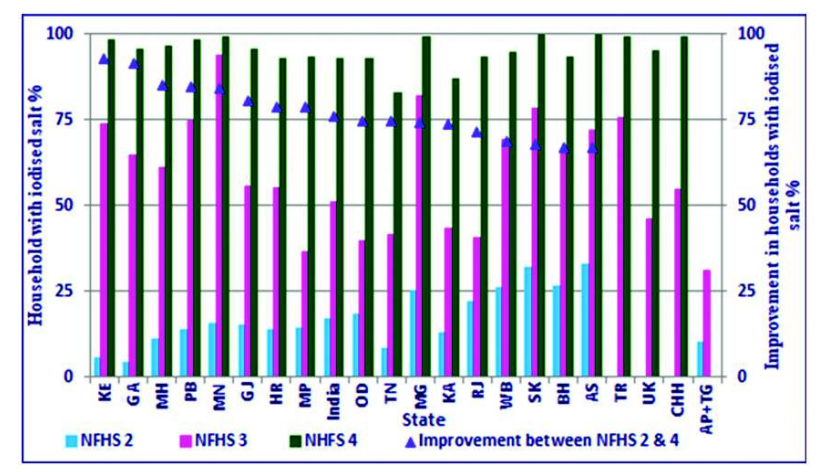

Fig. 4: Time trend in Household use of iodised salt (NFHS2, 3 , and 4) (copy right permission obtained from NFI Bulletin). Kerala (KE), Goa (GA), Maharashtra (MH), Punjab (PB), Manipur (MN), Gujarat (GJ), Haryana (HR), Madhya Pradesh (MP), India Odisha (OD), Tamil Nadu (TN), Meghalaya (MG), Karnataka (KA), Rajasthan (RJ), West Bengal (WB), Sikkim (SK), Bihar (BH), Assam (AS), Tripura (TR), Uttarakhand (UK), Chhattisgarh (CHH), Andhra Pradesh undivided (AP+TG) 
and Family Welfare and research studies on iodine levels at the manufacturer level, at the retailer level and household level and also study urinary excretion of iodine. Based on these data, a decision can be taken as to whether to continue with the present higher level of iodine fortification at the manufacturers' level.

Global as well as Indian studies have highlighted the linkage between salt intake and hypertension. Based on these findings, the WHO has recommended that the salt intake should be limited to $5 \mathrm{~g} /$ person/day (WHO 2012). In the last decade there have been several awareness generation efforts in India to promote reduction of salt intake from the current average of $10 \mathrm{~g} /$ person/day to $5 \mathrm{~g} /$ person/day. Available data from NNMB surveys (NNMB 2012) and research studies suggest that salt intake still continues to be between 8-12 g/day. The available data on salt intake from different regions in India have to be collated and reviewed to assess whether there has been any decline in salt intake. If salt intake has come down substantially, there will be a need to consider changes in the salt iodisation level.

With near-universal access to iodised salt there has been a substantial reduction in the prevalence of goitre. In this scenario, urinary iodine is the most useful indicator for assessing access to and use of iodised salt. In India there are no data on iodine content of water or food in different regions. These data are difficult to generate and monitor over time. In the absence of such data urinary iodine estimation can provide an indication of the total quantum of iodine intake of the population in different regions. There is an urgent need to ensure that urinary iodine testing is available in all state food testing laboratories, so that information on adequacy of salt iodisation can be monitored and appropriate mid-course corrections in the programme can be made. If urinary iodine in any region is persistently high, the factors responsible and health consequences arising from it have to be investigated.

\section{Fortification of Iodised Salt with Iron to Combat Iron Deficiency Anaemia}

Prevalence of anaemia in India is the highest in the world (Fig. 5). The prevalence of anaemia is high, not only among under-nourished poor persons but also in normal and over-nourished individuals from middle and upper income households. Low dietary intake of iron and folate is the major factor responsible for iron deficiency and folate deficiency and anaemia. In the last two decades there has been an increase in the reported prevalence of vitamin B 12 deficiency as well.

Given that iron deficiency is so wide spread, attempts have been made to fortify the universally consumed salt with iron. Salt also has the advantage

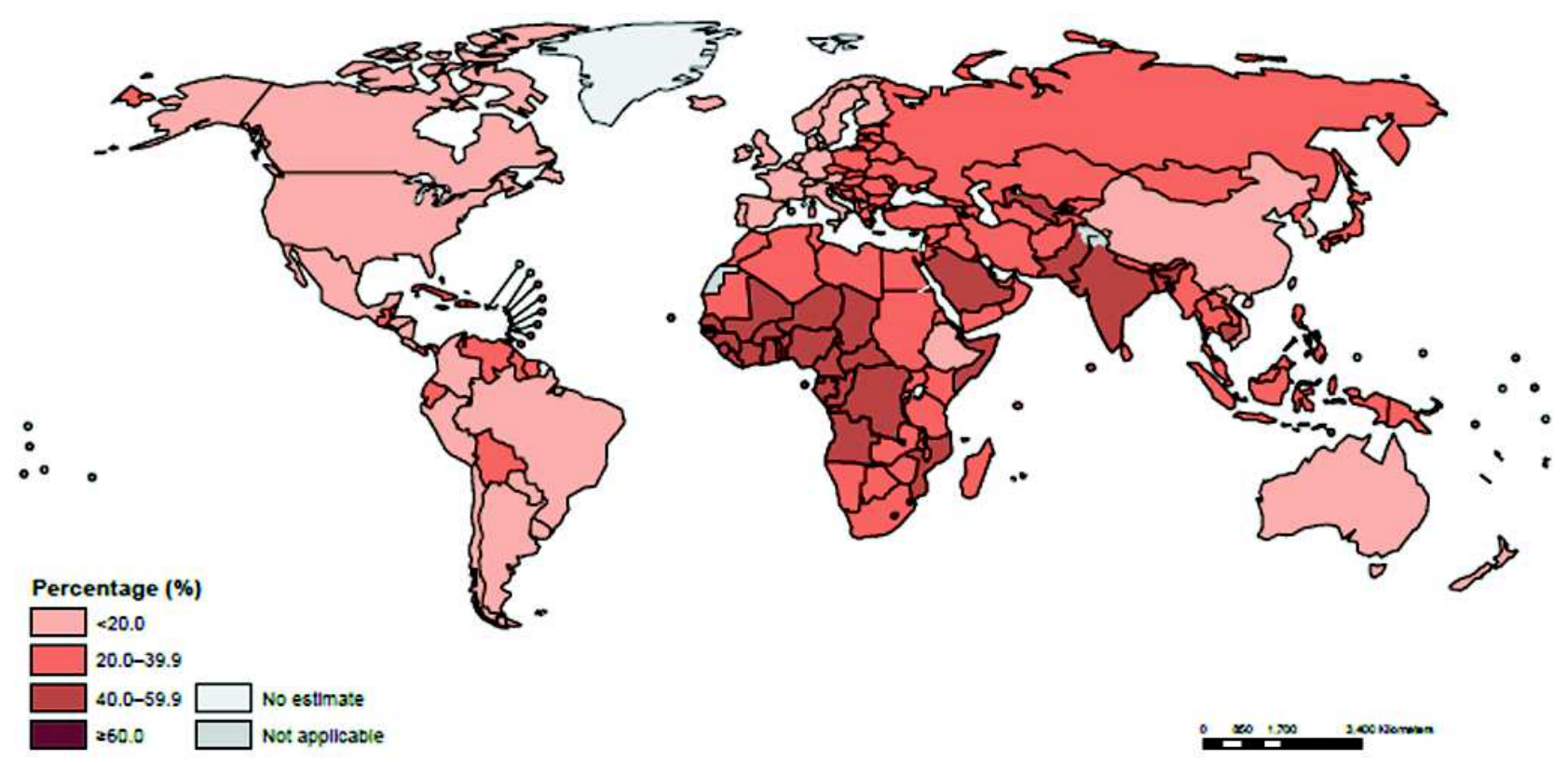

Fig. 5: Worldwide prevalence of anaemia in women aged 15-49 years (WHO 2011) 
that excessive consumption is not possible. A technology for fortifying salt with iodine and iron (DFS-Double fortified salt) has been developed by the National Institute of Nutrition and transferred to the industry (Sivakumar 2001, NIN 2005). Food Safety and Standards Authority of India (FSSAI) has approved two technologies for manufacture of ironfortified iodised salt. Voluntary fortification of salt using these technologies is under way. Compared to iodised salt, DFS costs more by Rs. $2-5 / \mathrm{kg}$. With increased production, DFS will become readily accessible, and with increased off-take the cost differential may come down. Guidelines were issued in 2011 for the mandatory use of DFS in hot cooked food in the MidDay Meal (MDM) programme and Integrated Child Development Services (ICDS) programme; however the states had difficulties in procuring DFS and so the coverage was very low. These guidelines have recently been re-issued. Implementation of these guidelines will ensure that the installed production capacity for DFS is fully utilised and awareness and acceptance of DFS is built up. DFS production, transport and supply can be rapidly upscaled using the centralised production and pre-existing programme for salt fortification with iodine. After ensuring adequate capacity for production, transport, off-take through Government programmes, supply through PDS and open market for home consumption, the process of legislating for mandatory fortification of salt with iron and iodine can begin. If the use of DFS in households is near-universal and sustained, it is possible to achieve population-wide increase in the intake of iron and achieve the World Health Assembly (WHA)/SDG target for reducing anaemia in women of reproductive age by 50\% (UNDP 2017).

\section{Fortification of Cereals with Iron, Folic Acid and Vitamin B12}

Fortification of cereals (rice and atta) with iron, folic acid and vitamin B12 has been advocated for combating anaemia. The FSSAI has notified the standards for rice and "atta" fortification with iron folic acid and vitamin B12. Voluntarily fortification of rice and wheat flour ("atta") with iron, folic acid and vitamin B12 is being taken up. Large-scale studies are currently underway to explore feasibility and the impact of using rice and "atta" fortified with iron, folic acid and vitamin B12. The Department of Women and Child Development has sent a communication to the state governments requesting them to use fortified "atta" for the hot cooked meal in MDM and ICDS programmes.

\section{Adverse Consequences of Excessive Intake of Iron}

In the last decade, potential adverse health consequences of excessive intake of iron have been documented. The difference between iron requirement in Indians $(17 \mathrm{mg} /$ day in men, $38 \mathrm{mg}$ /day in pregnant women) and the tolerable upper limit of iron (TUL $45 \mathrm{mg} /$ day) is low. If some segments of population who are currently consuming adequate iron and also begin to consume multiple food items that are fortified with iron, it is possible that TUL for iron may be exceeded. Experience with iron and folic acid (IFA) supplementation shows that minor gastrointestinal side effects are initially seen in $10 \%$ of persons who received iron supplements and because of this coverage under the programme was low. A similar situation can arise with the use of multiple food stuffs fortified with iron. In this context, it is preferable to concentrate the efforts on scaling up production of DFS and achieve universal access to it. After achieving universal access to DFS, iron may have to be removed from the fortification mixture for cereals in order to prevent the risk of the total intake of iron exceeding the TUL in some persons.

\section{Fortification of Wheat Flour with Folic Acid for Prevention of Neural Tube Defects}

It has been well documented that genetic polymorphisms affecting folate metabolism in the mother is associated with an increased risk of neural tube defects (NTD). Medical Research Council, UK, carried out a study on the effectiveness of periconceptional folic acid supplementation for prevention of the recurrence of NTD in the subsequent pregnancy. This study showed that there was a 79\% reduction in the recurrence of NTD in subsequent pregnancy following administration of high doses of folic acid (4 mg daily (MRC 1991). Subsequent studies have confirmed that folic acid supplementation does reduce NTD. Based on these findings, many countries initiated peri-conceptional folic acid supplementation especially in women with previous history of NTD. But compliance with peri-conceptional folic acid supplementation was low. In an effort to substantially reduce NTDs in the country, USA initiated mandatory 
fortification of wheat flour with folic acid. This resulted in a 19 to $32 \%$ reduction in the incidence of NTD in USA. Fortification of wheat did prevent some, but not all, NTD (Crider, 2011).

Since folic acid is a water-soluble vitamin it was assumed that in individuals with adequate dietary intake of folates, any excess intake following mandatory fortification will be excreted in urine. It has been assumed that fortification with water-soluble vitamins such as folic acid with large gap between RDA and TUL (TUL 1mg), will not lead to adverse health consequences (Crider, 2011). Almost all the data on TUL are derived from therapeutic supplementation of the nutrient alone or in combination, for a relatively short period of time under medical supervision. The applicability of findings from short term supplementation studies to mandatory fortification for indefinitely long periods has been debated. Areview of the situation in USA in the first decade after mandatory folic acid fortification of wheat flour concluded that excessive folate intake may mask vitamin B12 deficiency anaemia, precipitate or exacerbate neuropathy in vitamin B12-deficient individuals.

There were reports that folic acid supplementation may enhance the development and progression of already existing, undiagnosed premalignant and malignant lesions or increase the risk of cardiovascular diseases; but these have not been confirmed by other studies (Crider, 2011). However, these data have highlighted the need for close monitoring of all large-scale public health fortification programmes and periodical review of the risks and benefits associated with them. In India, in the ongoing voluntary fortification of rice and wheat flour, the three micro-nutrients, namely iron, folic acid and vitamin $\mathrm{B} 12$, are being incorporated at one third of their respective RDA levels. As folic acid, and vitamin B12 are used together there is no risk of masking vitamin B12 deficiency and incurring adverse health consequences.

\section{Fortification of Milk and Oil with Vitamins A and D}

Diet surveys carried out by NNMB have shown that the intake of Vitamin A is significantly lower than the RDA in young children, adolescent girls and pregnant women in India. Over the last three decades there has been a decline in clinical vitamin A deficiency in under-five children in the country but the biochemical deficiency of vitamin A continues to be common especially in children (Fig. 6).

Vitamin D is manufactured in skin exposed to sun light. India is a sun drenched country; but plasma vitamin D assays have shown that majority of Indians across all states, and all age, sex and economic groups

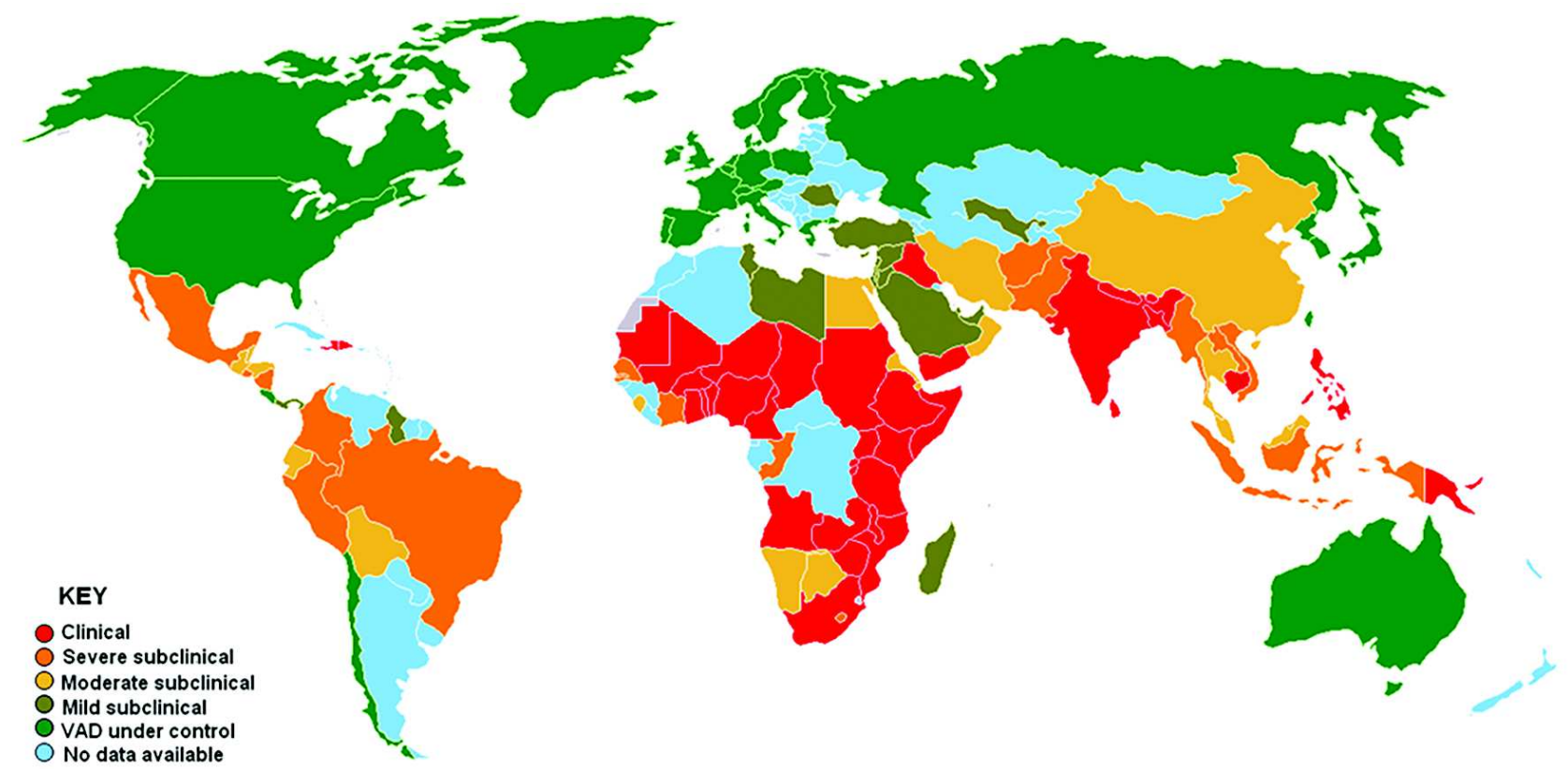

Fig. 6: Worldwide prevalence of vitamin A deficiency (WHO 1995) 


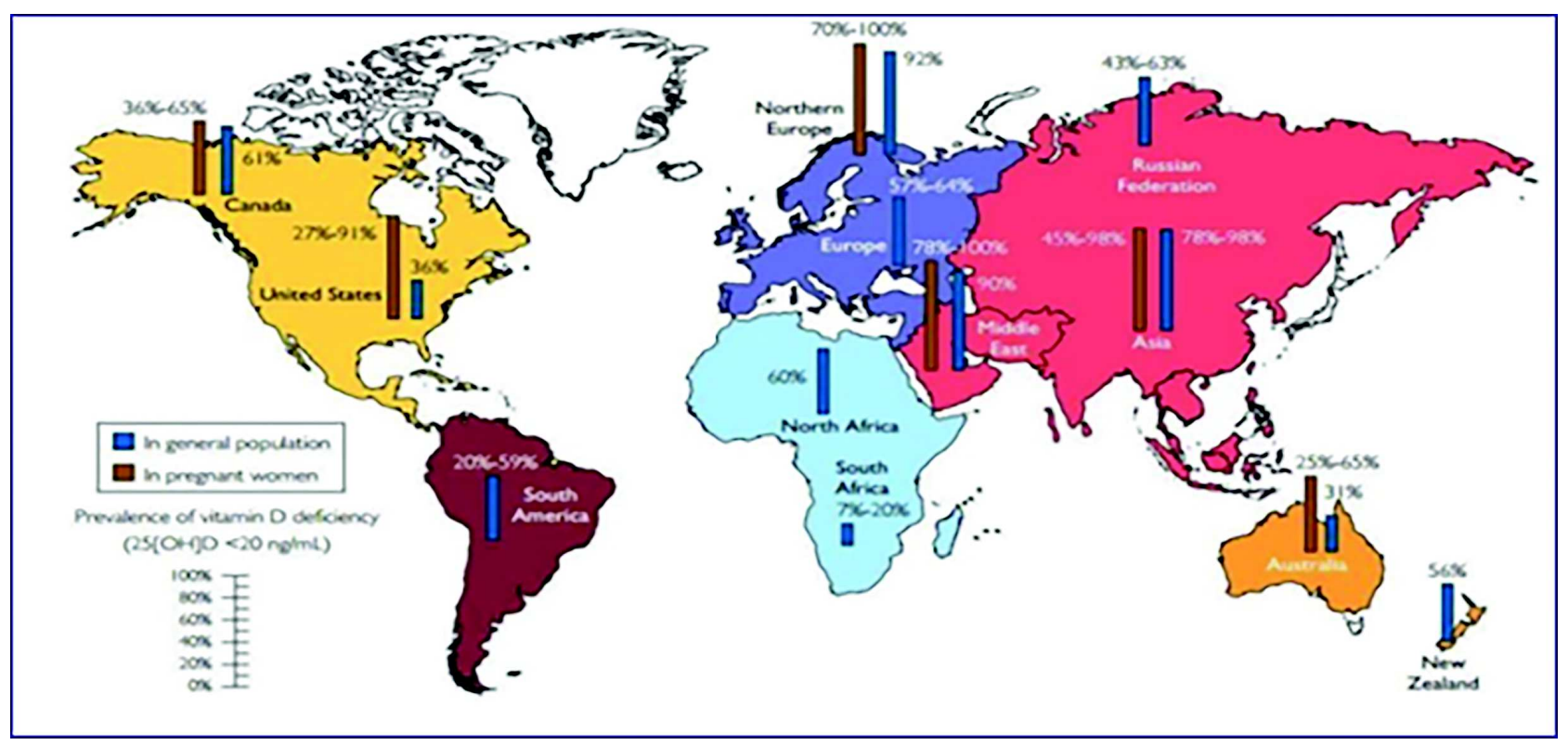

Fig. 7: Worldwide prevalence of Vitamin D Deficiency (Holick 2013)

have blood vitamin D levels indicative of deficiency (Fig. 7). Fortification of edible oils and milk with vitamins $A$ and $D$ is a simple, easy and inexpensive process. The FSSAI has notified the standards for fortifying vegetable oils with vitamins A and D. Voluntary fortification of edible oil is being taken up by some manufacturers. Feasibility, sustainability and efficacy of fortifying oil and milk with vitamins A and $\mathrm{D}$ is being explored.

White revolution has resulted in India becoming the number 1 milk producer in the world. Average milk production is $300 \mathrm{ml} / \mathrm{per}$ capita and this is projected to increase to $350 \mathrm{ml} /$ day by 2020 . About $20 \%$ of milk is processed in organized sector. The standards for fortification of milk with vitamins A and D have been notified by the FSSAI. Some of the largescale milk producers have voluntarily started fortification of toned, double-toned and skimmed milk with these vitamins.

\section{Multiple Micro-nutrient Fortification}

It is well documented that the diets of poorer segments of our population might be deficient in many micronutrients and several micro-nutrient deficiencies coexist in vulnerable segments of the population. In view of this, there have been efforts to fortify a variety of food stuffs with multiple micro-nutrients. In addition, micro-nutrient powders which could be added to home food prior to consumption have also been investigated (Best et al., 2011). A systematic review of these studies suggests that, though there may be some improvement in individual nutrient levels as shown by biochemical parameters after several months of consumption of fortified foods, such fortification may not reduce multiple micro-nutrient deficiencies, and there were no substantial health benefits (Eilander, 2011). In segments of the population who have adequate intake of one or more nutrients from food, the consumption of multiple food stuffs fortified with multiple nutrients, may result in a higher than required intake of some nutrients. Excessive intake of nutrients or imbalance of intake between nutrients due to multiple micro-nutrient fortification of multiple food stuffs may have adverse health consequences.

\section{Summary and Conclusion}

Micro-nutrient deficiencies, especially those of iodine, iron, folate, vitamin B 12, vitamin A and vitamin D are major public health problems in many segments of the population in India. Through fortification of appropriate food stuff, it is possible to achieve sustained improvement in the intake of these micro-nutrients and reduction in micro-nutrient deficiencies at the population level. India's effort to eliminate IDD through universal salt iodisation has resulted in over $90 \%$ of households using iodised salt and a steep decline in IDD. Globally as well as in India, iron 
deficiency is the most common micro-nutrient deficiency and is the major cause of anaemia. DFS represents the most feasible, economical and sustainable method of increasing iron intake by an average of $10 \mathrm{mg} /$ day and reducing the prevalence of iron deficiency and anaemia. Centralised production of edible salt and the pre-existing programme for fortification of salt with iodine, offers a ready platform to launch iron-fortified iodised salt and raises the hope that the country will be able to scale up access to DFS and achieve the SDG target of 50\% reduction in the prevalence of anaemia in women. Voluntary fortification of vegetable oils and milk with vitamins

\section{References}

Best C, Neufingerl N, Del Rosso J M ,Transler C, van den Briel $\mathrm{T}$ and Osendarp S (2011) Can multi-micronutrient food fortification improve the micronutrient status, growth, health, and cognition of schoolchildren? A systematic review Nutrition Reviews 69 186-204

Crider K S, Lynn B, Bailey L B and Berry R J (2011) Folic Acid Food Fortification - Its History, Effect, Concerns, and Future Nutrients 3 370-384 https://www.ncbi.nlm.nih.gov/ pmc/articles/PMC3257747 accessed on 22.6.2018

de Benoist B, McLean E, Andersson M, Rogers L (2008) Iodine deficiency in 2007: Global progress since 2003 Food Nutr Bull 29 195-202

DGHS (2018) National Iodine Deficiency Disorders Control Programme - dghs.gov.in/content/1348_3_NationalIodine Deficiency.aspx accessed on 22.6. 2018

Eilander A, Gera T, Sachdev H P S, Henk C T van der Knaap C M, Frans J Kok F J, Osendarp S J M (2010) Multiple micronutrient supplementation for improving cognitive performance in children: systematic review of randomized controlled trials Am J Clin Nutr 91 115-30

Food and Drug Administration (1996) Food standards: amendment of standards of identity for enriched grain products to require addition of folic acid Fed Reg 61 8781-8797

FAO World Food Summit 1996-www.fao.org/WFS/ accessed on 22.6. 2018

NHM (2016) Guidelines - National Horticulture Mission midh.gov.in/nhm/Guidline.htm accessed on 22.6. 2018

Hossein-nezhad A and Holick M F (2013) Vitamin D for Health: A Global Perspective Mayo Clin Proc Jul 88 720-755

IIPS: National Family Health Survey (NFHS) 2 - http://rchiips.org/ $\mathrm{nfhs} / \mathrm{nfhs} 2 . \mathrm{shtml}$ accessed on 22.6.2018

IIPS: National Family Health Survey (NFHS) 3 http://rchiips.org/ nfhs/nfhs3.shtml accessed on 22.6. 2018
$\mathrm{A}$ and $\mathrm{D}$ is underway.

Food fortification does represent a sustainable method of increasing the population's intake for selected micro-nutrients. However, fortification of multiple food stuff with multiple micro-nutrients is not advisable because of the potential adverse health consequences of excessive intake of some micronutrients and/or imbalance of intake of inter-related micro-nutrients by segments of the population who are already consuming one or more of these nutrients in sufficient amount in their daily diets.

IIPS: National Family Health Survey (NFHS) 4 Fact sheets http:/ /rchiips.org/nfhs/factsheet_NFHS-4.shtml

Medical Research Council (1991) Prevention of neural tube defects: results of the Medical Research Council Vitamin Study. MRC Vitamin Study Research Group Lancet 338 131-137

NIN (2005) Double fortified salt as a tool to control iodine deficiency disorders and iron deficiency anaemia - A report National Institute of Nutrition, Hyderabad NNMB (2012) National Nutrition Monitoring bureau repeat surveys -

nnmbindia.org/1_NNMB_Third_Repeat_Rural_Survey_ Technicl_Report_26.pdf accessed on 22.6. 2018

Sivakumar B, Brahmam G N V, Madhavan Nair K, Ranganathan S, Vishnuvardhana Rao M, Vijayaraghavan K, Kamala Krishnaswamy (2001) Prospects of fortification of salt with iron and iodine British Journal of Nutrition 5 S167S173

UNDP (2017) The Sustainable Development Goals Report https://unstats.un.org/sdgs/files/.../TheSustainable DevelopmentGoalsReport2017.pdf accessed on 22.6.2018

WHO (1995) Global prevalence of Vitamin A Deficiency www.who.int/nutrition/publications/micronutrients/ vitamin_a_deficiency/WHO.../en accessed on 26.6.2018

WHO (2006) Guidelines on food fortification with micronutrients www.who.int/nutrition/publications/micronutrients/ 9241594012/enaccessed on 22.6. 2018

WHO (2011) Use of multiple micronutrient powders for home fortification of foods whqlibdoc.who.int/publications/ 2011/9789241502047_eng.pdf accessed on 22.6. 2018

WHO (2011) The global prevalence of anaemia in 2011 www.who.int/nutrition/publications/.../global_prevalence_ anaemia_2011/en/accessed on 26.6.2018

WHO (2012) Guideline: Sodium intake for adults and children www.who.int/nutrition/publications/guidelines/ sodium_intake_printversion.pdf accessed on 25.6.2018. 